\title{
Referenzgruppeneffekte auf die Lernmotivation in den Fächern Mathematik und Biologie an Schweizer Gymnasien
}

\section{Stephan Schumann, Maren Oepke und Franz Eberle}

In der im Querschnitt angelegten Studie werden Referenzgruppeneffekte auf vier Facetten der Lernmotivation in den Fächern Mathematik und Biologie an Deutschschweizer Gymnasien mittels Mehrebenenanalysen untersucht $(N=2426)$. In Mathematik können für die intrinsische Motivation, das Interesse und die Leistungsmotivation Wirkungen auf Klassenebene nachgewiesen werden, in Biologie hingegen nur für letztere. Für die langfristig-instrumentelle Motivation kann kein solcher Fischteich-Effekt identifiziert werden. Weiterhin zeigt sich für das Fach Mathematik, dass die Effekte für die Facetten der Lernmotivation unterschiedlich stark ausfallen. Die Ergebnisse sprechen somit sowohl im Hinblick auf die Domäne als auch bezüglich der Facetten der Lernmotivation für eine differentielle Betrachtungsweise. Theoretische Implikationen werden diskutiert.

\section{Einleitung}

Wenn Schülerinnen oder Schüler ihre eigenen schulischen Kompetenzen einschätzen, so hängt diese Beurteilung nicht nur von vorhandenen individuellen Fähigkeiten, sondern auch von den Fähigkeiten von Mitschülerinnen und Mitschülern ab (Trautwein \& Lüdtke, 2010). Dieser als Referenz- bzw. Bezugsgruppeneffekt bezeichnete Sachverhalt ist insbesondere im Hinblick auf das schulische Selbstkonzept gut untersucht. Prominent sind dabei Studien zum so genannten Fischteich-Effekt (Big-Fish-Little-Pond-Effect, BFLPE). Der BFLPE beschreibt unter der Annahme gleicher individueller Fähigkeiten folgenden Zusammenhang: Je leistungsschwächer die Mitschülerinnen und Mitschüler sind (little pond), desto höher ist das Selbstkonzept (big fish) und umgekehrt (Marsh, 1987; Marsh et al., 2008).

In jüngerer Zeit wird in verschiedenen Arbeiten auf die Notwendigkeit hingewiesen, Studien zu Referenzgruppeneffekten über das Selbstkonzept hinaus auf weitere Variablen wie z.B. die Lernmotivation verstärkt auszudehnen (Marsh et al., 2008; Trautwein \& Lüdtke, 2010). Die Erforschung solcher Bezugsgrup- 
peneffekte auf die Lernmotivation befindet sich jedoch erst in den Anfängen. Köller, Daniels, Schnabel und Baumert (2000), Köller, Trautwein, Lüdtke und Baumert (2006) sowie Trautwein, Lüdtke, Marsh, Köller und Baumert (2006) können Referenzgruppeneffekte für das Interesse im Fach Mathematik nachweisen. Weitgehend unklar ist, ob sich derartige Fischteich-Effekte auch im Hinblick auf weitere Facetten der Lernmotivation zeigen. Darüber hinaus sollte untersucht werden, inwieweit motivationale Effekte in anderen Schulfächern identifiziert werden können (Köller et al., 2006; Trautwein \& Lüdtke, 2010).

An diesen Fragen setzt der vorliegende Beitrag an. Wir untersuchen unter Bezugnahme auf den motivationstheoretischen Rahmen von Eccles et al. (1983), ob sich Referenzgruppeneffekte auf das Interesse, die intrinsische Motivation, die langfristig-instrumentelle Motivation und die Leistungsmotivation von Schülerinnen und Schülern am Ende der Gymnasialzeit nachweisen lassen. Um Domäneneffekte vergleichen zu können, wird neben Mathematik das Fach Biologie in die Analysen einbezogen.

\section{Befundlage zu Effekten des Leistungsniveaus von Mitschülerinnen und Mitschülern auf das schulische Selbstkonzept und weitere Variablen}

Die psychologischen Auswirkungen von Vergleichsprozessen innerhalb von Referenzgruppen sind schon länger Gegenstand der Forschung (Festinger, 1954; Kelley, 1952; Thibaut \& Kelley, 1959). Herbert Marsh und Kollegen beschreiben und analysieren seit Mitte der 1980er-Jahre u.a. unter Bezug auf Festingers (1954) Theorie sozialer Vergleichsprozesse und die soziologische Arbeit von Davis (1966) den eingangs dargestellten Fischteich-Effekt (Marsh, 2005; Marsh et al., 2008; Marsh \& Parker, 1984): Je leistungsschwächer die Mitschülerinnen und Mitschüler sind, desto höher ist - bei gleicher Leistung - das individuelle Selbstkonzept und umgekehrt. Trotz einiger kritischer Studien zur Gültigkeit des Fischteich-Effekts (Dai, 2004; Dai \& Rinn, 2008; Plucker et al., 2004) kann die Befundlage als robust bezeichnet werden: Der Effekt zeigt sich in einer Vielzahl an Studien für verschiedene Länder und für verschiedene Bildungsstufen einschliesslich der Gymnasialstufe (dazu näher Seaton, 2007). Der BFLPE wurde dabei vor allem im Hinblick auf das schulische (akademische) Selbstkonzept nachgewiesen. In einer noch überschaubaren Zahl an Untersuchungen wird deutlich, dass der Fischteich-Effekt nicht auf das schulische Selbstkonzept beschränkt ist. So zeigt er sich u.a. für das allgemeine Selbstkonzept, für Bildungsund Berufsaspirationen, für Kurswahlen, für Anstrengung und physische Aktivitäten (Marsh, 1991; Marsh \& O’Mara, 2010; Trautwein, Gerlach \& Lüdtke, 2008). Die Effekte auf diese Variablen werden dabei partiell oder vollständig über das schulische Selbstkonzept vermittelt (Marsh \& O'Mara, 2010). Insgesamt ist die Befundlage zu Effekten des Leistungsniveaus von Mitschülerinnen und Mitschülern auf Variablen jenseits des Selbstkonzepts jedoch dünn (Plucker et al., 2004; Trautwein et al., 2006; Trautwein \& Lüdtke, 2005). 
Aus der Perspektive des vorliegenden Beitrags von besonderem Interesse sind die Studien von Köller et al. (2006) und Trautwein et al. (2006), welche u.a. Referenzgruppeneffekte auf das fachliche Interesse in Mathematik untersuchen. Die Untersuchung von Köller et al. (2006) ist dabei auf die gymnasiale Oberstufe bezogen. Trautwein et al. (2006) untersuchen Lernende der neunten Jahrgangsstufe unter Verwendung der deutschen PISA-2000-Stichprobe (Baumert et al., 2002). In beiden Studien kann der Fischteicheffekt für das mathematische Interesse nachgewiesen werden. Die Effektstärken der auf Schulebene aggregierten Leistungen fallen jedoch sehr unterschiedlich aus und liegen ohne Kontrolle weiterer Variablen bei -0.12 ( $\mathrm{p}<.05$ ) (Köller et al., 2006) bzw. bei $-0.62(\mathrm{p}<.001)$ (Trautwein et al., 2006). ${ }^{1}$ Bei alleiniger Betrachtung von Schülerinnen und Schülern an Gesamtschulen finden Trautwein et al. (2006) eine Effektstärke von -0.08 ( $\mathrm{p}<.05$ ). Unter Kontrolle des Schultyps, der Noten und vor allem des Selbstkonzepts verschwindet in beiden Studien der Referenzgruppeneffekt auf das Interesse.

\section{Theoretische Annäherungen an die Wirkung Leistungs- bezogener Referenzgruppeneffekte auf verschiedene Konstrukte der Lernmotivation}

Nachdem verschiedene Studien Referenzgruppeneffekte für das fachliche Interesse für Mathematik nachgewiesen haben (s.o.), ist zu fragen, ob sich diese Wirkungen über das Interesse hinaus auch für weitere Motivationskonstrukte zeigen und wie solche Effekte theoretisch erklärt werden können. Die Überlegungen im vorliegenden Beitrag beziehen sich auf vier unterschiedliche Konstrukte der Lernmotivation: auf die intrinsische Motivation, die Leistungsmotivation, auf den langfristig wahrgenommenen Nutzen der fachlichen Lerninhalte sowie im Sinne einer Replikation oben genannter Befunde auf das fachliche Interesse. Die Konstrukte entstammen prominenten Motivationstheorien und deren Anwendung auf schulische Kontexte: Theorien der intrinsischen Motivation, Zieltheorien, Erwartungs-Wert-Theorien und Interessentheorien (einen Überblick geben Eccles \& Wigfield, 2002; Pintrich \& Schunk, 2002). Jenseits der Tatsache, dass diese Theorien jeweils eigene historisch-konzeptuelle Entwicklungsbezüge aufweisen, existieren untereinander verschiedene konzeptuelle Anknüpfungsmöglichkeiten. So legen Eccles et al. (1983) mit ihrem Erwartungs-Wert-Modell ein umfassendes Modell vor, welches verschiedene Motivationsperspektiven verschränkt (vgl. Abbildung 1). ${ }^{2}$

Wie andere Motivationstheorien auch versucht das Modell menschliches Verhalten durch den subjektiven Wert der Verhaltenskonsequenz zu erklären. Unter Bezugnahme auf pädagogische Kontexte postulieren Eccles et al. (1983), dass die Motivation von Lernenden und die Selektion der Aufgaben, welche sie angehen, direkt von den damit verbundenen Erfolgserwartungen und dem subjektiven Wert, den sie der Aufgabe zumessen, beeinflusst wird. Auf diese Komponenten gibt es verschiedene indirekte Einflüsse wie zum Beispiel das sozio-kulturelle Milieu. 


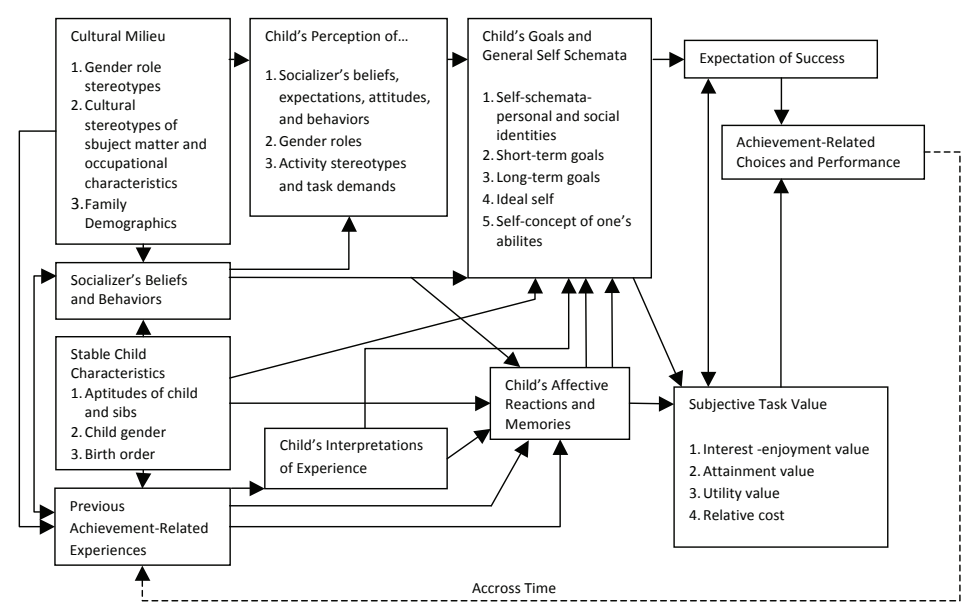

Abbildung 1: Erwartungs-Wert-Modell nach Eccles et al. (1983) (aus: Eccles \& Wigfield, 2002)

Das Eccles-Modell kann - obwohl es originär für Schülerinnen und Schüler im Kindesalter entwickelt wurde - auch für die im vorliegenden Beitrag vorgenommene theoretische Annäherung herangezogen werden, da es den subjektiv wahrgenommenen Wert von fachlichen Anforderungen (subjective task value) im Sinne der oben genannten Motivationskonstrukte ausdifferenziert (Eccles \& Wigfield, 2002). Der Wertaspekt gliedert sich dabei in einen intrinsic ${ }^{3}$ and interest value, einen attainment value und einen utility value zuzüglich eines wahrgenommenen psychologischen Kostenaspekts, welcher hier nicht näher betrachtet wird.

Mit diesen Wertkomponenten wird explizit Bezug auf Konstrukte anderer Motivationstheorien genommen (Eccles \& Wigfield, 2002). Während der intrinsic value vor allem die wahrgenommene Freude an einer Lernhandlung umfasst und damit weitgehend dem Konzept der intrinsischen Motivation entspricht (Deci \& Ryan, 1985), entsteht das Interesse (interest value) in erster Linie aufgrund einer wahrgenommenen aktuellen persönlichen Relevanz der Lern- und Aufgabeninhalte (Hidi \& Renninger, 2006; Schiefele, Krapp \& Winteler, 1992). Der utility-Komponente sind Anknüpfungspunkte zu Formen extrinsischer Motivation inhärent, die auf kurz- und langfristige Ziele ausgerichtet ist (Eccles \& Wigfield, 2002). Dabei bestehen insbesondere mit der identifizierten Regulationsform der Selbstbestimmungstheorie der Motivation erhebliche Überschneidungen (Deci \& Ryan, 1985). Bei dieser Motivationsform erfolgt die Bewertung einer aktuellen Anforderung einschliesslich ihrer erfolgreichen Bewältigung aus deren angenommener persönlichen Relevanz für die Zukunft (z.B. für Karriereziele; Eccles \& Wigfield, 2002). Der attainment value ist nach Eccles et al. (1983) die wahrgenommene Wichtigkeit, bei einer Aufgabe gut abzuschneiden (Battle, 1966). Hier liegt der Bezug zu vorliegenden 
Zieltheorien auf der Hand (Ramseier, 2004). Prominente Zieltheorien unterscheiden zunächst dichotom zwischen einer Aufgabenorientierung (mastery goals) und einer Ich-Orientierung (performance goals; Ames, 1992). Performance goals werden zudem häufig nochmals in performance-approach goals und performance-avoidance goals unterteilt (Elliot \& Church, 1997; Midgley et al., 1998). Im vorliegenden Beitrag wird im empirischen Teil nur auf performance-approach goals Bezug genommen. Diese kennzeichnen eine Orientierung, bei der die erfolgreiche Bewältigung einer Aufgabe angestrebt wird, um eine gute Leistung zu zeigen. Als Vergleichsstandard dient bei der Einschätzung der eigenen Fähigkeiten vor allem auch die relative Position zu Mitschülerinnen und Mitschülern in der Bezugsgruppe, d.h. in der Regel in der Klasse (Midgley et al., 1998; Nicholls, 1984). Damit fließt in das Konstrukt der performance-approach goals explizit das Konzept des Referenzgruppenvergleichs ein.

Im vorliegenden Beitrag wird mit Bezug auf das Eccles-Modell folgende Terminologie verwendet: intrinsische Motivation (intrinsic value) und Interesse (interest value), Leistungsmotivation (performance-approach goals als eine Ausprägung des attainment value) und langfristig-instrumentelle Motivation (utility value).

Die Fragestellung unseres Beitrags lautet im Kern, ob für diese vier Lernmotivationskonstrukte Referenzgruppeneffekte identifiziert werden können und welche Erklärungsansätze sich hierfür anbieten. Trautwein et al. (2006) verweisen unter Bezug auf das Eccles-Modell auf die Mediator-Rolle des schulischen Selbstkonzepts. Eccles und Wigfield (2002) argumentieren, dass diese Effekte vor allem für ältere Schülerinnen und Schüler zu erwarten sind, während bei Jüngeren die Konstrukte noch unabhängig voneinander seien. Schaut man allerdings in die einschlägige motivationspsychologische Literatur, so ist die Befundlage bezüglich der postulierten kausalen Ordnung uneindeutig. Denn obwohl viele Motivationstheorien mehr oder weniger explizit von einer Beeinflussung der Lernmotivation durch Kompetenzüberzeugungen bzw. das Selbstkonzept ausgehen, zeigen sich in den wenigen vorliegenden Längsschnittstudien zumeist keine signifikanten Effekte (Nurmi \& Aunola, 2005; Obach, 2003; Skaalvik \& Valaas, 1999; Spinath \& Spinath, 2005). Jacobs, Lanza, Osgood, Eccles und Wigfield (2003) sowie Marsh, Craven, Hinkley und Debus (2003) können dagegen kleine Effekte nachweisen. Jacquelynne Eccles, die in ihrem Modell von einer Beeinflussung durch das Selbstkonzept ausgeht, kommentiert die uneinheitliche Befundlage mit der Feststellung: «Thus, it seems very likely that, ..., learning and task motivation, competence levels and perceived competence levels are integrally connected. We do not understand how the processes start and how they interact with each other» (Eccles, 2005, S. 111).

Spinath und Spinath (2005) führen als eine Erklärung für die uneinheitliche Befundlage an, dass ein Einfluss des Selbstkonzepts auf die Lernmotivation vor allem dann zu erwarten sei, wenn Schülerinnen und Schüler soziale Vergleichsprozesse innerhalb der Bezugsgruppe anstellen und intraindividuelle, temporale 
Vergleiche eine eher untergeordnete Rolle spielen. Trifft diese Feststellung $\mathrm{zu}$, so würde dies bedeuten, dass für die hier untersuchte Leistungsmotivation besonders stark ausgeprägte Fischteich-Effekte zu erwarten sind.

\section{Fragestellungen}

In der vorliegenden Studie wird der Einfluss des durchschnittlichen Leistungsniveaus von gymnasialen Schulklassen auf vier verschiedene Konstrukte der Lernmotivation (Interesse, intrinsische Motivation, langfristig-instrumentelle Motivation, Leistungsmotivation) untersucht. In die Analysen wird neben Mathematik das Fach Biologie einbezogen.

Zunächst wird der Frage nachgegangen, welchen Einfluss das durchschnittliche Klassenleistungsniveau auf die verschiedenen Motivationskonstrukte unter Kontrolle der Individualleistung, des Geschlechts und der Wahl des gymnasialen Schwerpunktfachs (Kurswahl) hat. Schülerinnen und Schüler wählen ein Schwerpunktfach, in welchem sie vertiefte Kenntnisse erwerben. Laut dem gültigen Maturitätsanerkennungs-Reglement (MAR 95, 1995) stehen acht Schwerpunktfächer zur Auswahl (u.a. "Physik und Anwendungen der Mathematik» sowie «Biologie und Chemie»). Bislang liegen kaum Befunde zu Referenzgruppeneffekten auf über das Interesse an Mathematik hinaus gehende Facetten der Lernmotivation vor. Wie oben gezeigt, erlauben zudem die vorliegenden motivationstheoretischen Modelle nur bedingt Annahmen zu diesem Einfluss. Aus theoretischer Sicht sind in erster Linie Bezugsgruppeneffekte für die Leistungsmotivation erwartbar. Zudem gehen wir für die Vorhersage des Mathematik-Interesses von einer Replikation der Befunde von Köller et al. (2006) und Trautwein et al. (2006) aus. Angemerkt wird dabei, dass in unserer Studie das Selbstkonzept nicht erfasst wurde, so dass die Prüfung einer Mediatorrolle dieses Merkmals nicht möglich ist.

Im Hinblick auf die Domänenspezifität wird zudem untersucht, ob bezüglich der Referenzgruppeneffekte systematische Unterschiede zwischen den Fächern Mathematik und Biologie bestehen. Im Unterschied zum Fach Mathematik liegen zu Biologie kaum Befunde zu Referenzgruppeneffekten vor. Daher wird auch auf eine Formulierung von Hypothesen verzichtet.

\section{Methode}

Die Datengrundlage für die vorliegende Untersuchung liefert die 2007 in der deutsch- und französischsprachigen Schweiz durchgeführte Studie "Evaluation der Maturitätsreform 1995 Phase II (EVAMAR II)». Ziel von EVAMAR II war es, die Studierfähigkeit von Maturandinnen und Maturanden in der Schweiz zu erfassen. Die querschnittliche Erhebung fand am Ende des letzten Schuljahres 
im Gymnasium statt. Zum Einsatz kamen Tests und ein Schülerfragebogen. Details zu Hintergrund, Methodik und Resultaten von EVAMAR II können bei Eberle et al. (2008) nachgeschlagen werden.

\section{Stichprobe}

Die in der vorliegenden Untersuchung verwendeten Daten basieren auf dem Deutschschweizer Teil der Stichprobe, da die Items zur Lernmotivation ausschliesslich in dieser Sprachregion erfasst wurden.

Nach Behandlung der fehlenden Werte befinden sich 2‘426 Fälle im Datensatz. Die Stichprobe besteht zu 58\% aus Schülerinnen und zu 42\% aus Schülern. Die Schülerinnen und Schüler befanden sich im letzten Gymnasialjahr (überwiegend 12. Jahrgangsstufe, in einzelnen Kantonen 13. Jahrgangsstufe) kurz vor dem Ablegen der Maturaprüfungen und waren im Mittel 18 Jahre alt. Die Stichprobe umfasste 148 Klassen, welche wiederum 67 Schulen entstammten. In den Klassen befanden sich im Durchschnitt 17 Schülerinnen und Schüler. Das Schwerpunktfach «Physik und Anwendungen der Mathematik» bzw. «Biologie und Chemie» belegten 9\% bzw. 11\% der Jugendlichen. Die Stammklassen sind teilweise schwerpunktgemischt.

Im Unterschied zu einigen anderen Studien zum Fischteich-Effekt werden in unserer Studie Schulklassen und nicht Schulen als Referenzgruppen verwendet. Dies kommt der Realität von im schulischen Kontext stattfindenden Vergleichsprozesse näher (Köller et al., 2006; Skaalvik \& Skaalvik, 2002).

\section{Instrumente}

Standardisierte Mathematik- und Biologieleistungstests: Die Tests wurden in der Studie EVAMAR II entwickelt. Die Anforderungen wurden aus den Studienmaterialien und ersten Zwischenprüfungen der 16 gemessen an den Studierendenzahlen grössten universitären Studienfächer der Schweiz abgeleitet. Der Mathematiktest enthält vor allem Aufgaben zu Inhalten, die für eine breite Anzahl an Studienfächern und nicht speziell für das Studienfach Mathematik von Bedeutung sind. Inhaltlich lassen sich die Items den Bereichen "Analysis», "Geometrie», «Stochastik» und «Vermischtes» zuordnen. Im Haupttest kamen insgesamt 175 Items zum Einsatz. Diese Items wurden im Rahmen eines Multi-Matrix-Designs auf sechs Testhefte mit rotierenden Aufgabenblöcken verteilt. Dabei kamen folgende Aufgabenformate zum Einsatz: Multiple-Choice-Aufgaben, Aufgaben mit mehreren Behauptungen sowie offene Aufgaben mit eindeutigen Lösungen. Die mathematische Fähigkeit ist eindimensional repräsentiert und weist eine EAP/PV-Reliabilität von 0.81 auf.

Der Biologietest hat den Charakter eines Wissenstests zu Fachinhalten, die vor allem für ein Biologie- oder Medizinstudium, aber auch teilweise für nicht-naturwissenschaftliche Studien wichtig sind. Sie sind in den Inhaltsbereichen «Evolution», «Struktur und Funktion» (Cytologie, Anatomie), «Genetik», "Informationsverarbeitung, Verhalten und Immunbiologie», "Ökologie» und 
«Stoffwechsel» angesiedelt. Die insgesamt $126 \mathrm{im}$ Test eingesetzten Items waren im Rahmen des Multi-Matrix-Designs auf sieben Testhefte mit rotierenden Aufgabenblöcken verteilt. Einige wenige Items hatten ein offenes Format, die mit einem biologischen Fachbegriff beantwortet werden konnten, die übrigen Items wiesen ein Multiple-Choice-Format mit vier Antwortoptionen auf. Auch für den Biologietest erwies sich eine eindimensionale Lösung als empirisch sinnvoll. Die EAP/PV-Reliabilität beträgt 0.66.

Insgesamt prüfen der Mathematik- und der Biologietest Wissen, welches an Schweizer Universitäten vorausgesetzt und in der Schule vermittelt wird, der mathematische Testteil erfasst zudem mathematisches Verständnis.

Motivationale Variablen: Es wurden vier unterschiedliche Konstrukte der fachspezifischen Lernmotivation erfasst: intrinsische Motivation, fachliches Interesse, Leistungsmotivation sowie die langfristig-instrumentelle Motivation. Die Itemformulierungen sind jeweils auf Mathematik bzw. Biologie ausgerichtet und wurden vierstufig erfasst $(1=$ trifft nicht $\mathrm{zu}, 2=$ trifft eher nicht $\mathrm{zu}, 3$ $=$ trifft eher zu, $4=$ trifft völlig zu). Die Itemformulierungen können in ihrer Zugehörigkeit zum jeweiligen Konstrukt aus dem Anhang entnommen werden. Ersichtlich werden dabei auch die Quellen, an welchen sich die Operationalisierungen anlehnen. Die Reliabilitätskennwerte (Cronbachs alpha) für die acht Skalen liegen in einem Bereich von 0.79 bis 0.92 .

Weitere Variablen: In die Analysen wurden zudem die Geschlechtszugehörigkeit und die Wahl des Schwerpunktfachs (SPF) einbezogen. Die Variable «Schwerpunktfach» unterscheidet dabei dichotom für den Bereich Mathematik zwischen «anderes SPF = 1» und «SPF Physik und Anwendungen der Mathematik = 2» sowie für den Bereich Biologie zwischen «anderes SPF $=1$ » und «SPF Biologie und Chemie = 2». Für die Analysen im Bereich Biologie erfolgte zudem die Berücksichtigung einer Variablen «Fach abgeschlossen». An vielen Schweizer Gymnasien wird dieses Fach ein oder vereinzelt zwei Jahre vor der Maturität abgeschlossen. Die Variable ist dichotom definiert («abgeschlossen = 1» und «Teilnahme bis Maturitätsprüfung = 2»). In der untersuchten Stichprobe haben $76 \%$ der Lernenden das Fach abgeschlossen.

\section{Statistische Analysen}

Skalierung der Leistungstests: Die Daten der beiden Leistungstests wurden im Rahmen der EVAMAR II-Analysen auf der Basis der Item-Response-Theorie (IRT) ausgewertet, die jeweils fünf «plausible values» für die Personenfähigkeiten auf einen Mittelwert für die gesamte Stichprobe von 500 Punkten (SD: 100 Punkte) standardisiert sowie daraus das arithmetische Mittel gebildet.

Mehrebenenmodellierung: Im vorliegenden Beitrag steht der Einfluss der individuellen sowie der auf Klassenebene aggregierten Mathematik- bzw. Biologie-Leistung auf die Varianten der Lernmotivation im Fokus des Interesses. Das übliche Vorgehen beinhaltet dabei eine Modellierung der individuellen Leistung der Lernenden auf der ersten Ebene (erwarteter positiver Effekt 
höherer individueller Leisungen) und eine Modellierung der Klassenleistung (Referenzgruppe) auf der zweiten Ebene (erwarteter negativer Effekt höherer aggregierter Leistungen). Um die beiden Ebenen in den empirischen Auswertungen angemessen zu berücksichtigen, wurden Mehrebenen-Regressionsanalysen (Random-Intercept-Modelle) mittels des Statistik-Programms MPlus durchgeführt (Muthén \& Muthén, 2007). Auf Ebene 2 wurden die beobachteten Klassenmittelwerte aus der Stammklasse verwendet. Die Zentrierung der Prädiktoren auf der ersten Ebene erfolgte mittels der Option Grandmean Centering (Enders \& Tofighi, 2007; Trautwein \& Lüdtke, 2008).

Behandlung fehlender Werte: Im Bereich der Mathematik fehlte zu 4.5\% der Fälle ein Personenfähigkeitswert. In Biologie traf dies auf 5.7\% der Probanden zu. Die fehlenden Werte sind auf Nicht-Teilnahme an diesen Testteilen zurückzuführen. Sie wurden aus sachlogischen Gründen nicht ersetzt.

Im Bereich der motivationalen Variablen existierten je Skala zwischen 5.5\% bis $8.2 \%$ fehlender Werte. Trotz dieser eher geringen Ausfallquote wurden für diese Variablen Ersetzungen mittels mutipler Imputationen vorgenommen. Hierfür wurde das Statistik-Programm LatentGold 4.5 (Vermunt \& Magidson, 2005) verwendet. Es wurden 48 Imputationsdatensätze erzeugt und von diesen der erste verwendet.

\section{Ergebnisse}

\section{Deskriptive Statistik und bivariate Zusammenhänge}

Tabelle 1 zeigt die deskriptive Statistik für die motivationalen Konstrukte und für die Testleistungen in Mathematik und Biologie. Zudem ist jeweils der Intraklassenkoeffizient (ICC) zur Identifikation der Varianz auf der Ebene 2 (Klassenebene) angegeben. Dabei wird erstens deutlich, dass die Leistungsstreuung auf der Klassenebene in Mathematik mit 0.28 doppelt so gross ist wie in Biologie (0.14). Die ICC-Werte zeigen zweitens, dass im Bereich der domänenspezifischen fachlichen Kompetenzen die Varianzen auf Klassenebene grösser sind als im Bereich der motivationalen Variablen. Letztere bewegen sich in einer Bandbreite von 0.03 bis 0.13 und liegen in einem für motivationale Variablen üblichen Rahmen (vgl. z.B. die ICC-Werte in Trautwein et al., 2006).

Die deskriptiven Ergebnisse verweisen darauf, dass in Biologie die Schülerinnen und Schüler über eine hohe intrinsische Motivation verfügen und an den Inhalten interessiert sind. Die Items zur instrumentellen und zur leistungsbezogenen Motivation erfahren vergleichsweise weniger Zustimmung. In Bezug auf das Fach Mathematik liegen die Ausprägungen der vier Motivationskonstrukte näher beieinander. 
Tabelle 1: Deskriptive Statistik

\begin{tabular}{lrrrrrrrrr}
\hline & \multicolumn{3}{c}{ Mathematik } & & \multicolumn{4}{c}{ Biologie } \\
\cline { 2 - 3 } Variable & ICC & M & SD & & ICC & M & SD \\
\hline Testleistung & 0.28 & 499.16 & 89.23 & & 0.14 & 515.86 & 79.90 \\
Interesse & 0.08 & 1.86 & 0.70 & & 0.11 & 2.65 & 0.74 \\
Intrinsische Motivation & 0.10 & 2.13 & 0.78 & & 0.13 & 2.70 & 0.75 \\
Langfristig-instrumentelle Motivation & 0.10 & 1.96 & 0.86 & & 0.07 & 1.98 & 0.88 \\
Leistungsmotivation & 0.03 & 2.14 & 0.74 & & 0.05 & 2.15 & 0.78 \\
\hline
\end{tabular}

Anmerkung: ICC = Intraklassenkorrelation (geschätzt mittels des Nullmodells in MPlus)

Die bivariaten Zusammenhänge zwischen den interessierenden Variablen sind in Tabelle 2 dargestellt. Die Interkorrelationen der berücksichtigten Variablen zeigen Folgendes: Die Testleistungen in Mathematik und Biologie weisen einen kleinen bis mittleren Zusammenhang auf (0.27). Wie in der Studie EVAMAR II beabsichtigt, ist es gelungen, unterschiedliche kognitiv-fachliche Bereiche der Studierfähigkeit abzubilden (Eberle et al., 2008). Die Zusammenhänge der Mathematik- bzw. der Biologieleistung zu den vier fachbezogenen Lernmotivationsfacetten sind durchgängig theoriekonform, wobei die Interkorrelationen für Biologie niedriger ausfallen als für Mathematik. Männliche Jugendliche weisen in beiden Testbereichen höhere Werte auf, wobei der Vorsprung in Mathematik deutlicher ausfällt. Dies korrespondiert mit der Befundlage zu geschlechtsspezifischen Differenzen im mathematisch-naturwissenschaftlichen Bereich im deutschsprachigen Raum (Köller \& Klieme, 2000; Ramseier, 2004). Auch der positive Zusammenhang der domänenspezifischen Testleistung zur Wahl des jeweiligen Schwerpunktfachs (Physik und Anwendungen der Mathematik bzw. Biologie und Chemie) konnte erwartet werden.

Die Zusammenhänge zwischen den Motivationsfacetten innerhalb der Domäne sind für beide Fächer durchgängig positiv. Sehr hoch ist dabei jeweils die Interkorrelation zwischen dem fachlichen Interesse und der intrinsischen Motivation (0.79 für Mathematik bzw. 0.81 für Biologie). Auf eine Zusammenführung zu einem gemeinsamen Faktor wird jedoch aus zwei Gründen verzichtet. Erstens entstammen die beiden Facetten unterschiedlichen theoretischen Ansätzen. Und zweitens erlaubt die gesonderte Berücksichtigung des fachlichen Interesses eine Replikation der Ergebnisse einschlägiger Studien zum BFLPE auf das Interesse (Köller et al., 2000; Köller et al., 2006; Trautwein et al., 2006).

Zwischen den jeweiligen domänenbezogenen motivationalen Pendants (z.B. Mathematik-Interesse und Biologie-Interesse) bestehen mit einer Ausnahme keine oder geringe Interkorrelationen. Einzig zwischen den beiden leistungsbezogenen Motivationsformen existiert ein grosser Zusammenhang (0.69). Dies ist erwartungskonform, da das zugrunde liegende Handlungsmotiv ich-orientiert und nicht inhalts- bzw. domänenbezogen konzeptualisiert ist (Ramseier, 2004). 


\begin{tabular}{|c|c|c|c|c|c|c|c|c|c|c|}
\hline & (1) & (2) & (3) & (4) & (5) & $(6)$ & $(7)$ & $(8)$ & (9) & (10) \\
\hline \multicolumn{11}{|l|}{ Testleistung Mathematik (1) } \\
\hline Testleistung Biologie (2) & .27 & & & & & & & & & \\
\hline Interesse Mathematik (3) & .36 & .11 & & & & & & & & \\
\hline $\begin{array}{l}\text { Intrinsische Motivation } \\
\text { Mathematik (4) }\end{array}$ & .35 & .07 & .79 & & & & & & & \\
\hline $\begin{array}{l}\text { Langfristig-instrumentelle } \\
\text { Motivati-on Mathematik (5) }\end{array}$ & .32 & .12 & .48 & .46 & & & & & & \\
\hline $\begin{array}{l}\text { Leistungsmotivation Mathe- } \\
\text { matik (6) }\end{array}$ & .11 & .03 & .29 & .33 & .23 & & & & & \\
\hline Interesse Biologie (7) & $-.05^{*}$ & .26 & $.05^{*}$ & $-.02^{+}$ & $-.02^{+}$ & .13 & & & & \\
\hline $\begin{array}{l}\text { Intrinsische Motivation } \\
\text { Biologie (8) }\end{array}$ & $-.06^{*}$ & .25 & .03 & $-.01^{+}$ & $.01^{+}$ & .10 & .81 & & & \\
\hline $\begin{array}{l}\text { Langfristig-instrumentelle } \\
\text { Motivation Biologie (9) }\end{array}$ & $-.02^{+}$ & .20 & $.05^{*}$ & $-.01^{+}$ & .15 & .14 & .46 & .49 & & \\
\hline $\begin{array}{l}\text { Leistungsmotivation } \\
\text { Biologie (10) }\end{array}$ & $-.02^{+}$ & .08 & $.06^{*}$ & $.04^{*}$ & $.07^{*}$ & .69 & .32 & .30 & .30 & \\
\hline Geschlecht (11) & -.23 & -.10 & -.18 & -.11 & -.22 & .12 & .24 & .22 & .11 & .16 \\
\hline $\begin{array}{l}\text { Schwerpunktfach Ma bzw. } \\
\text { Bio (12) }\end{array}$ & .42 & .31 & .26 & .24 & .28 & $.00^{+}$ & .16 & .17 & .27 & $.05^{*}$ \\
\hline Biologie abgeschlossen (13) & - & .26 & - & - & - & - & .18 & .18 & .25 & .09 \\
\hline
\end{tabular}

Anmerkungen: Signifikanzniveaus: ${ }^{*} \mathrm{p}<0.05,{ }^{+} \mathrm{p}>0.05$, alle anderen: $\mathrm{p}<0.01$, Kodierungen: Geschlecht ( 1 = männlich, 2 = weiblich), Schwerpunktfach $(1=$ anderes SPF, $2=$ SPF Physik und Anwendungen der Mathematik bzw. SPF Biologie und Chemie), Biologie abgeschlossen ( $1=$ abgeschlossen, $2=$ Teilnahme bis Maturitätsprüfung)

Im Hinblick auf die Geschlechtseffekte lässt sich feststellen, dass mit Ausnahme der leistungsbezogenen Motivation im Bereich mathematikbezogener Lernmotivationsfacetten höhere Werte für die männlichen Schüler beobachtet werden können, während in Biologie durchgängig die Schülerinnen die höheren Werte aufweisen. Dieser zweite Befund ist bemerkenswert, da die Schülerinnen im Bereich der Biologieleistung trotz höherer Lernmotivation die geringeren Testscores erzielten. Die Wahl des jeweiligen Schwerpunktfachs spiegelt sich schliesslich - mit Ausnahme der Leistungsmotivation - in höheren Werten der Lernmotivation wieder.

\section{Beantwortung der Fragestellungen}

Die zentrale Frage im vorliegenden Beitrag richtet sich auf den Einfluss des durchschnittlichen Klassenleistungsniveaus auf vier verschiedene Motivationskonstrukte. Dabei erfolgt eine Differenzierung zwischen den Fächern Mathematik und Biologie. Die Tabellen 3 (Mathematik) und 4 (Biologie) zeigen die Ergebnisse für die entsprechenden Modelle. Auf der Individualebene wurde in 
den Analysen jeweils die Individualleistung, das Geschlecht und die Wahl des Schwerpunktfachs sowie im Falle von Biologie die Variable «Fach abgeschlossen» kontrolliert.

Tabelle 3: Ergebnisse der Mehrebenenanalysen zu den motivationalen Referenzgruppeneffekten im Fach Mathematik

\begin{tabular}{|c|c|c|c|c|c|c|c|c|}
\hline \multirow[b]{3}{*}{ Prädiktoren } & \multicolumn{8}{|c|}{ Motivationsart } \\
\hline & \multicolumn{2}{|c|}{ Interesse } & \multicolumn{2}{|c|}{$\begin{array}{l}\text { Intrinsische } \\
\text { Motivation }\end{array}$} & \multicolumn{2}{|c|}{$\begin{array}{l}\text { Langfristig- } \\
\text { instrumentelle } \\
\text { Motivation }\end{array}$} & \multicolumn{2}{|c|}{$\begin{array}{l}\text { Leistungs- } \\
\text { motivation }\end{array}$} \\
\hline & $b$ & $S E$ & $b$ & $S E$ & $b$ & $S E$ & $b$ & $S E$ \\
\hline \multicolumn{9}{|l|}{ Individualebene } \\
\hline Geschlecht & $-.10^{* * *}$ & .02 & -.04 & .02 & $-.13^{* * *}$ & .02 & $-.14^{* * *}$ & .02 \\
\hline Leistung (individuell) & $.31^{* * *}$ & .02 & $.35^{* * *}$ & .02 & $.23^{* * *}$ & .02 & $.19^{* * *}$ & .03 \\
\hline Schwerpunktfach & $.15^{* * *}$ & .03 & $.15^{* * *}$ & .03 & $.16^{* * *}$ & .03 & .00 & .03 \\
\hline \multicolumn{9}{|l|}{ Klassenebene } \\
\hline Leistung (aggregiert) & $-.37^{*}$ & .15 & $-.40^{* *}$ & .12 & -.08 & .19 & $-.58^{* * *}$ & .14 \\
\hline \multicolumn{9}{|l|}{ Erklärte Varianz } \\
\hline Individualebene & .19 & .02 & .19 & .02 & .15 & .02 & .04 & .01 \\
\hline Klassenebene & .14 & .11 & .16 & .09 & .01 & .03 & .33 & .16 \\
\hline
\end{tabular}

Anmerkungen: Signifikanzniveaus: ${ }^{*} \mathrm{p}<0.05,{ }^{* *} \mathrm{p}<0.01,{ }^{* * *} \mathrm{p}<0.001$, Kodierungen: Geschlecht $(1=$ männlich, 2 = weiblich), Schwerpunktfach $(1=$ anderes SPF, 2 = SPF Physik und Anwendungen der Mathematik),

Zunächst interessiert, ob der aus anderen Studien bekannte Referenzgruppeneffekt auf das mathematische Interesse repliziert werden kann. Aus Tabelle 3 geht hervor, dass der Effekt auch in der vorliegenden Studie identifiziert werden kann $(b=-0.37)$. Zugleich macht Tabelle 4 deutlich, dass dieser Effekt auf das Interesse im Fach Biologie nicht nachgewiesen werden kann.

Mit Blick auf die drei anderen Facetten der Lernmotivation zeigt sich für das Fach Mathematik folgendes Bild: Im Hinblick auf die intrinsische Motivation ist der Referenzgruppeneffekt mit $b=-0.40$ sogar noch etwas grösser als für das fachliche Interesse. Für die langfristig-instrumentelle Lernmotivation kann kein Fischteich-Effekt identifiziert werden. Der grösste Effekt lässt sich schliesslich für die Leistungsmotivation beobachten $(b=-0.58)$.

Dieser Befund kommt nicht überraschend, da sich die Leistungsmotivation theoretisch explizit über soziale Vergleichsprozesse erklären lässt. Die Tatsache, dass in der vorliegenden Studie mit 33\% der deutlich grössere Teil der Varianz auf Klassenebene erklärt werden kann, unterstreicht diese Annahme.

Im Fach Biologie kann ausschliesslich für die Leistungsmotivation ein Referenzgruppeneffekt identifiziert werden. Dieser fällt mit $b=-0.32$ allerdings geringer als im Fach Mathematik aus. Zudem liegt die erklärte Varianz auf 
Klassenebene bei nur 11\%. Für die drei anderen Formen der Lernmotivation können auf der Klassenebene keine Wirkungen überzufällig abgesichert werden.

Tabelle 4: Ergebnisse der Mehrebenenanalysen zu den motivationalen
Referenzgruppeneffekten im Fach Biologie

\begin{tabular}{|c|c|c|c|c|c|c|c|c|}
\hline \multirow[b]{3}{*}{ Prädiktoren } & \multicolumn{8}{|c|}{ Motivationsart } \\
\hline & \multicolumn{2}{|c|}{ Interesse } & \multicolumn{2}{|c|}{$\begin{array}{l}\text { Intrinsische } \\
\text { Motivation }\end{array}$} & \multicolumn{2}{|c|}{$\begin{array}{l}\text { Langfristig- } \\
\text { instrumentelle } \\
\text { Motivation }\end{array}$} & \multicolumn{2}{|c|}{$\begin{array}{l}\text { Leistungs- } \\
\text { motivation }\end{array}$} \\
\hline & $b$ & $S E$ & $b$ & $S E$ & $b$ & $S E$ & $b$ & $S E$ \\
\hline \multicolumn{9}{|l|}{ Individualebene } \\
\hline Geschlecht & $.28^{* * *}$ & .02 & $.26^{* * *}$ & .02 & $.14^{* * *}$ & .02 & .17 & .02 \\
\hline Leistung (individuell) & $.24^{* * *}$ & .02 & $.24^{* * *}$ & .02 & $.13^{* * *}$ & .03 & .11 & .03 \\
\hline Schwerpunktfach & .03 & .04 & .04 & .04 & $.15^{* * *}$ & .04 & .01 & .04 \\
\hline Fach abgeschlossen & $.17^{* * *}$ & .03 & $.18^{* * *}$ & .04 & $.16^{* * *}$ & .03 & .09 & .03 \\
\hline \multicolumn{9}{|l|}{ Klassenebene } \\
\hline Leistung (aggregiert) & .01 & .13 & -.01 & .13 & -.10 & .19 & -.32 & .14 \\
\hline \multicolumn{9}{|l|}{ Erklärte Varianz } \\
\hline Individualebene & .18 & .02 & .18 & .02 & .13 & .02 & .05 & .01 \\
\hline Klassenebene & .00 & .00 & .00 & .00 & .01 & .04 & .11 & .09 \\
\hline
\end{tabular}

Anmerkungen: Signifikanzniveaus: ${ }^{*} \mathrm{p}<0.05,{ }^{* *} \mathrm{p}<0.01,{ }^{* * *} \mathrm{p}<0.001$, Kodierungen: Geschlecht ( 1 = männlich, 2 = weiblich), Schwerpunktfach ( 1 = anderes SPF, 2 = SPF Biologie und Chemie), Biologie abgeschlossen ( 1 = abgeschlossen, 2 = Teilnahme bis Maturitätsprüfung)

\section{Diskussion}

Die vorliegende Studie untersucht Bezugsgruppeneffekte auf verschiedene Facetten der Lernmotivation in den Fächern Mathematik und Biologie an Deutschschweizer Gymnasien. Die Ergebnisse sprechen in doppelter Hinsicht für eine differentielle Betrachtungsweise: Erstens wird deutlich, dass die untersuchten Effekte in erheblichem Masse domänenabhängig sind. So können für drei der vier motivationalen Konstrukte im Bereich der Mathematik Wirkungen auf der Klassenebene nachgewiesen werden. Dagegen lassen sich in Biologie für die intrinsische Motivation und das Interesse keine Effekte identifizieren. Es ist ausschliesslich ein Effekt für die Leistungsmotivation in Biologie beobachtbar. Berücksichtigen muss man jedoch, dass die Leistungen auf der Klassenebene im Bereich Biologie weniger stark streuen als in Mathematik. Zweitens zeigt sich an den Befunden zum Fach Mathematik, dass die Bezugsgruppeneffekte für die vier untersuchten Facetten der Lernmotivation sehr unterschiedlich ausfallen. Während für die Leistungsmotivation ein Grossteil der erklärten Varianz auf der Klassenebene verortet werden kann, spielt die Referenzgruppe für die Ausprägung der langfristig-instrumentellen Lernmotivation - wie in Biologie 
- keine Rolle. Mittlere Effektstärken lassen sich für die intrinsische Motivation und das fachliche Interesse beobachten. Damit werden zugleich die Befunde von Köller et al. (2000), Köller et al. (2006) sowie von Trautwein et al. (2006) zu Fischteich-Effekten auf das Interesse an Mathematik bestätigt.

Das im Beitrag verwendete motivationstheoretische Modell von Eccles et al. (1983) bietet sich als Rahmenmodell für die Einordnung des Forschungsgegenstandes an (Trautwein et al., 2006). So sind mit der intrinsischen Motivation und dem Interesse (intrinsic and interest value), der Leistungsmotivation (attainment value) und der langfristig-instrumentellen Motivation (utility value) alle hier untersuchten Lernmotivationsfacetten im Sinne von Wertkomponenten im Eccles-Modell vereint. Zudem werden diese Motivationsfacetten von Eccles in eine abhängige Beziehung zum Selbstkonzept gestellt, was insofern bedeutsam ist, als dass das Selbstkonzept die zentrale abhängige Variable in den meisten BFLPE-Studien ist. Zudem konnte in weiteren Studien zum Fischteich-Effekt auf andere Outcome-Variablen die vermittelnde Rolle des von uns nicht erfassten Selbstkonzepts empirisch nachgewiesen werden.

Die hier vorgelegten Ergebnisse verweisen vor diesem Hintergrund allerdings auf die oben erwähnten differentiellen Effekte, die durch das Eccles-Modell nur bedingt erklärt werden können. Einzig für die Leistungsmotivation lassen sich in beiden Fächern grosse Bezugsgruppeneffekte nachweisen. Dies ist erwartungskonform, da in die individuelle Ausprägung der Leistungsmotivation die relative Position zu Mitschülerinnen und Mitschülern in der Klasse mit einfliesst. Zugleich ist die genaue Wirkungsweise dieses Effekts offen. Eine Verknüpfung der Leistungsmotivationsforschung mit der Social Comparison Theory, die Marsh und Kollegen ihren BFLPE-Studien zugrunde legten (Seaton, 2007), steht hier noch aus. Wheeler und Suls (2005, S. 567) bemerken hierzu: «It is strange that Social Comparison Theory has not been better integrated into the achievement motivation literature». Dies ist nicht zuletzt auch damit begründet, dass sich die SCT nicht sonderlich detailliert mit den motivationalen Hintergründen sozialer Vergleichsprozesse auseinandersetzt (Seaton, 2007).

Aus theoretischer Perspektive bleibt auch offen, warum im Bereich Mathematik Referenzgruppeneffekte für die intrinsische Motivation und das fachliche Interesse auftreten, im Fach Biologie jedoch nicht. Das Eccles-Modell liefert hier keine Aufklärung, da Referenzrahmeneffekte im Modell keine Rolle spielen. Auch die einschlägigen Theorien der intrinsischen Motivation und des Interesses liefern für diesen Befund kaum Erklärungsansätze. Hier bieten sich weitere Untersuchungen an, die möglichst im Längsschnitt durchzuführen sind und die domänenspezifische Selbstkonzeptvariablen berücksichtigen. In der vorliegenden Studie sind diese beiden Punkte nicht erfüllt, was ohne Zweifel zentrale Limitationen der Untersuchung sind. Zugleich eröffnen die hier vorgelegten Befunde eine ganze Reihe weiterer Fragen, die hier nur angerissen werden konnten. 


\section{Anmerkungen}

$1 \mathrm{Zu}$ beachten ist bei der Interpretation der in Köller et al. (2006) und Trautwein et al. (2006) berichteten Effekte, dass es sich jeweils um unstandardisierte Regressionskoeffizienten handelt. Trautwein et al. (2006) begegnen dieser Problematik durch eine z-Transformation der betrachteten, kontinuierlichen Variablen. In Köller et al. (2006) wurde aufgrund des längsschnittlichen Charakters der Studie die z-Transformation nur für die Messwerte zum ersten Messzeitpunkt vorgenommen, die Werte zum zweiten Messzeitpunkt unterscheiden sich hiervon leicht. Die Höhe der in den beiden Studien berichteten Effekte ist folglich nur bedingt miteinander vergleichbar.

2 Auch Trautwein et al. (2006) verweisen im Hinblick auf eine Erklärung des BFLPE auf das Interesse an Mathematik auf dieses Modell.

3 Der Begriff des intrinsic value entspricht im Eccles-Modell dem enjoyment-value.

\section{Literatur}

Ames, C. (1992). Classrooms: Goals, structures, and student motivation. Journal of Educational Psychology, 84, 261-271.

Battle, E. S. (1966). Motivational determinants of academic competence. Journal of Personality and Social Psychology, 4, 634-642.

Dai, D. Y. (2004). How universal is the big-fish-little-pond effect? American Psychologist, 59, 267-268.

Dai, D. Y. \& Rinn, A. N. (2008). The big fish little pond effect: What do we know and where do we go from here? Educational Psychology Review, 20, 283-317.

Davis, J. A. (1966). The campus as a frog pond: An application of the theory of relative deprivation to career decisions of college men. American Journal of Sociology, 72, 17-31.

Deci, E. L. \& Ryan, R. M. (1985). Intrinsic motivation and self-determination in human behaviour. New York: Plenum Press.

Eberle, F., Gehrer, K., Jaggi, B., Kottonau, J., Oepke, M. \& Pflüger, M. (2008). Evaluation der Maturitätsreform 1995. Schlussbericht zur Phase II. Bern: Staatssekretariat für Bildung und Forschung.

Eberle, F., Schumann, S., Oepke, M., Müller, C., Barske, N., Pflüger, M. \& Hesske, S. (2009). Instrumenten- und Skalendokumentation zum Forschungsprojekt "Anwendungs- und problemorientierter Unterricht in gymnasialen Lehr-/Lernumgebungen (APU)». Universität Zürich: Institut für Gymnasial- und Berufspädagogik.

Eccles J. S. (2005). Subjective task value and the Eccles et al. model of achievement-related choices. In A. J. Elliot \& C. S. Dweck (Ed.), Handbook of competence and motivation (pp. 105-121). New York: The guilford press.

Eccles, J. S. \& Wigfield, A. (2002). Motivational beliefs, values, and goals. Annual review of Psychology, 53, 109-132.

Eccles, J. S., Adler, T. F., Futterman, R., Goff, S. B., Kaczala, C. M., Meece, J. L. \& Midgley, C. (1983). Expectations, values and academic behaviors. In J. T. Spence (Ed.), Perspectives on achievement and achievement motivation (pp. 75-146). San Francisco: Freeman.

Elliot, A. J. \& Church, M. A. (1997). A hierarchical model of approach and avoidance achievement motivation. Journal of Personality and Social Psychology, 72, 218-232.

Enders, C. K. \& Tofighi, D. (2007). Centering predictor variables in cross-sectional multilevel models: A new look at an old issue. Psychological Methods, 12, 121-138.

Festinger, L. (1954). A theory of social comparison processes. Human Relations, 7, 117-140.

Hidi, S. \& Renninger, K. A. (2006). The four-phase model of interest development. Educational Psychologist, 41, 111-127.

Jacobs, J. E., Lanza, S., Osgood, D. W., Eccles, J. S. \& Wigfield, A. (2003). Changes in children's self-competence and values: Gender and domain differences across grades one through twelve. Child Development, 73, 509-527. 
Kelley, H. H. (1952). The two functions of reference groups. In G. E. Swanson, T. M. Newcomb \& E. L. Hartley (Ed.), Readings in social psychology (pp. 410-414). New York: Holt.

Köller, O., Daniels, Z., Schnabel, K. \& Baumert, J. (2000). Kurswahlen von Mädchen und Jungen im Fach Mathematik: Zur Rolle von fachspezifischem Selbstkonzept und Interesse. Zeitschrift für Pädagogische Psychologie, 14, 26-37.

Köller, O. \& Klieme, E. (2000). Geschlechtsdifferenzen in den mathematisch-naturwissenschaftlichen Leistungen. In J. Baumert, W. Bos \& R. Lehmann (Hrsg.), TIMSS/III. Dritte Internationale Mathematik- und Naturwissenschaftsstudie. Mathematische und naturwissenschaftliche Bildung am Ende der Schullaufbahn (S. 373-404). Opladen: Leske und Budrich.

Köller, O., Trautwein, U., Lüdtke, O. \& Baumert, J. (2006). Zum Zusammenspiel von schulischer Leistung, Selbstkonzept und Interesse in der gymnasialen Oberstufe. Zeitschrift für Pädagogische Psychologie, 20, 27-39.

MAR 95. (1995). Verordnung des Bundesrates/Reglement der EDK über die Anerkennung von gymnasialen Maturitätsausweisen (MAR) vom 16. Januar/15. Februar 1995. Bern: Schweizerische Konferenz der kantonalen Erziehungsdirektoren (EDK).

Marsh, H. W. (1987). The big-fish-little-pond effect on academic self-concept. Journal of Educational Psychology, 79, 280-295.

Marsh, H. W. (1991). The failure of high-ability high schools to deliver academic benefits: The importance of academic self-concept and educational aspirations. American Educational Research Journal, 28, 445-480.

Marsh, H. W. (2005). The big-fish-little-pond effect on academic self-concept. Zeitschrift für Pädagogische Psychologie, 19, 119-128.

Marsh, H. W., Craven, R. G., Hinkley, J. W. \& Debus, R. L. (2003). Evaluation of the big-two-factor theory of academic motivation orientation: An evaluation of jingle-jangle fallacies. Multivariate Behavioral Research, 38, 189-224.

Marsh, H. W. \& O'Mara, A. J. (2010). Long-term total negative effects of school-average ability on diverse educational outcomes. Direct and indirect effects of the Big-fishlittle-pond effect. Zeitschrift für Pädagogische Psychologie, 24 (1), 51-72.

Marsh, H. W. \& Parker, J. W. (1984). Determinants of student self-concept: Is it better to be a relatively large fish in a small pond even if you don't learn to swim as well? Journal of Personality and Social Psychology, 47, 213-231.

Marsh, H. W., Seaton, M., Trautwein, U., Lüdtke, O., Hau, K. T., O`Mara, A. J. \& Craven, R. G. (2008). The Big-fish-little-pond-effect stands up to critical scrutiny: Implications for theory, methology, and future research. Educational Psychology Review, 20, 319-350.

Midgley, C., Kaplan, A., Middleton, M., Maehr, M., Urdan, T., Anderman, L. H., Anderman, E. \& Roeser, R. (1998). The development and validation of scales assessing students' achievement goal orientations. Contemporary Educational Psychology, 23, 113-131.

Muthén, L. K. \& Muthén, B. O. (2007). Mplus User's Guide. Los Angeles: Muthén \& Muthén.

Nicholls, J. G. (1984). Achievement motivation: Conceptions of ability, subjective experience, task choice, and performance. Psychological Review, 91, 328-346.

Nurmi, J. E. \& Aunola, K. (2005). Task-motivation during the first school years. A person-oriented approach to longitudinal data. Learning and Instruction, 15, 103-122.

Obach, M. S. (2003). A longitudinal-sequential study of perceived academic competence and motivational beliefs for learning among children in middle school. Educational Psychology, 23, 323-338.

Pintrich, P. R. \& Schunk, D. H. (2002). Motivation in education. Upper Saddle River: Merill Prentice Hall.

Plucker, J. A., Robinson, N. M., Greenspon, T. S., Feldhusen, J. F., McCoach, D. B. \& Subotnik, R. F. (2004). It's not how the pond makes you feel, but rather how high you can jump. American Psychologist, 59, 268-269. 
Prenzel, M., Kirsten, A., Dengler, P., Ettle, R. \& Beer, T. (1996). Selbstbestimmt motiviertes und interessiertes Lernen in der kaufmännischen Erstausbildung. Zeitschrift für Berufsund Wirtschaftspädagogik, 13, 108-127.

Ramseier, E. (2004). Motivation als Ergebnis und als Determinante schulischen Lernens. Eine Analyse im Rahmen von TIMSS. Unveröffentlichte Dissertation, Universität Zürich.

Schiefele, U., Krapp, A. \& Winteler, A. (1992). Interest as a predictor of academic achievement: A meta-analysis of research. In K. A. Renninger, S. Hidi \& A. Krapp (Ed.), The role of interest in learning and development (pp. 183-212). Hillsdale, NJ: Erlbaum.

Seaton, M. (2007). The big-fish-little-pond effect under the grill: Tests of its universality, a search for moderators, and the role of social comparasion. Unpublished doctoral dissertation, University of Western Sydney, Australia.

Skaalvik, E. M. \& Skaalvik, G. C. (2002). Internal and external frames of reference for academic self-concept. Educational Psychologist, 37, 233-244.

Skaalvik, E. M. \& Valas, H. (1999). Relations among achievement, self-concept, and motivation in mathematics and language arts: A longitudinal study. The Journal of Experimental Education, 67, 135-146.

Spinath, B. \& Spinath, F. M. (2005). Longitudinal analysis of the link between learning motivation and competence beliefs among elementary school children. Learning and Instruction, 15, 87-102.

Thibaut, J. W. \& Kelley, H. H. (1959). The social psychology of groups. New York: Wiley.

Trautwein, U., Gerlach, E. \& Lüdtke, O. (2008). Athletic classmates physical self-concept, and free-time physical activity: A longitudinal study of frame of reference effects. Journal of Educational Psychology, 100, 988-1001.

Trautwein, U. \& Lüdtke, O. (2005). The Big-Fish-Little-Pond Effect. Future research questions and educational implications. Zeitschrift für Pädagogische Psychologie, 19, 137-140.

Trautwein, U. \& Lüdtke, O. (2008). Predicting homework motivation and homework effort in six school subjects: The role of person and family characteristics, classroom factors, and school track. Learning and Instruction, 19, 243-258.

Trautwein, U. \& Lüdtke, O. (2010). Referenzgruppeneffekte. In W. Bos, E. Klieme \& O. Köller (Hrsg.), Schulische Lerngelegenheiten und Kompetenzentwicklung (S. 11-30). Münster: Waxmann.

Trautwein, U., Lüdtke, O., Marsh, H. W., Köller, O. \& Baumert, J. (2006). Tracking, grading, and student motivation: Using group composition and status to predict self-concept and interest in ninth-grade Mathematics. Journal of Educational Psychology, 98, 788-806.

Vermunt, J. K. \& Magidson, J. (2005). Latent GOLD 4.5 User's Guide. Belmont, MA: Statistical Innovations Inc.

Wheeler, L. \& Suls, J. (2005). Social comparison and self-evaluations of competence. In A. J. Elliot \& C. S. Dweck (Ed.), Handbook of Competence and Motivation (pp. 566-578). New York, London: The guilford press.

Schlagworte: Referenzgruppen, Motivation, Gymnasium, Mathematik, Biologie 


\section{Effets du groupe de référence sur la motivation pour les études dans les matières mathématique et biologie dans les lycées suisses}

\section{Ré sumé}

La présente étude examine, sur la base d'une analyse multidimensionnelle appliquée à un échantillon représentatif $(\mathrm{N}=2$ '426), les effets du groupe de référence sur quatre aspects de la motivation à l'étude des mathématiques et de la biologie dans des lycées de Suisse alémanique. En mathématiques, la motivation intrinsèque, l'intérêt et la motivation à la performance (Leistungsmotivation) sont identifiés comme influençant le niveau général de la classe; alors qu'en biologie, seul le dernier aspect intervient. En ce qui concerne la motivation à long terme, aucun effet n'a été mis en évidence. Il apparait en outre que, dans le domaine des mathématiques, les effets de ces facettes de la motivation se manifestent de manière différenciée. Ces résultats plaident en faveur d'une prise en compte des domaines disciplinaires aussi bien que des facettes de la motivation à apprendre dans une approche différentielle. Les applications théoriques de ces résultats font l'objet d'une discussion.

Mots-clés: Effets du groupe de référence, motivation, lycées, mathématique, biologie

\section{Effetti di un gruppo di riferimento sulla motivazione all'apprendimento in matematica e biologia nei licei svizzeri}

\section{Riassunto}

In questo lavoro si studiano gli effetti di un gruppo di riferimento su quattro aspetti della motivazione in matematica e biologia nei ginnasi della svizzera tedesca attraverso un'analisi multidimensionale $(\mathrm{N}=2$ ‘426). In matematica è possibile identificare degli effetti a livello della classe sulla motivazione intrinseca, sull'interesse e sulla motivazione alla risultato; in biologia invece solo l'ultimo effetto. Nessun effetto viene invece riscontrato per le due discipline sulla motivazione a lungo termine. Inoltre, $\mathrm{i}$ dati indicano che, per la matematica, gli effetti per i quattro aspetti della motivazione si manifestano in maniera differente. I risultati portano a consoderare i settori disciplinari per sviluppare uno sguardo differenziato in relazione alla motivazione. Vengono discusse alcune implicazioni teoriche.

Parole chiave: Effetti dei gruppi di riferimento, motivazione, liceo, matematica, biologia 


\section{Reference Group Effects on Learning Motivation in Mathematics and Biology in Swiss Grammar Schools}

\section{Summary}

This cross-sectional study in Swiss grammar schools examines via multilevel-modelling reference group effects on four constructs of learning motivation in mathematics and biology $(\mathrm{N}=2.426)$. In mathematics, class level effects on intrinsic motivation, interest, and achievement motivation can be identified. However, they vary substantially in size. In contrast, for biology there is only an effect on achievement motivation to be observed. Yet, neither of the two subjects show an effect on longterm-instrumental motivation. As a consequence thereof, future research should analyse reference group effects on learning motivation in schools with a more domain- and construct-specific perspective. Theoretical implications will be discussed.

Key words: Reference group effects, motivation, grammar school, mathematics, biology 


\section{Anhang}

\section{Itemformulierungen der vier Lernmotivationsskalen}

\begin{tabular}{|c|c|c|c|}
\hline Skala & & Items & Bezugsquellen \\
\hline & & $\begin{array}{l}\text { Im Mathematik/Biologie-Unter- } \\
\text { richt ... }\end{array}$ & \\
\hline \multirow[t]{3}{*}{ Intrinsische Motivation } & - & $\begin{array}{l}\text { macht das Lernen meistens Spass. } \\
\text { vergeht die Zeit häufig wie im } \\
\text { Flug. }\end{array}$ & $\begin{array}{l}\text { Eberle et al. (2009) } \\
\text { in Anlehnung an Prenzel et al. } \\
(1996)\end{array}$ \\
\hline & - & $\begin{array}{l}\text { ist der Unterrichtsstoff oft } \\
\text { spannend. }\end{array}$ & \\
\hline & - & $\begin{array}{l}\text { bin ich oft neugierig auf das, was } \\
\text { noch kommt. }\end{array}$ & \\
\hline \multirow[t]{3}{*}{ Interesse } & - & $\begin{array}{l}\text { stosse ich häufig auf interessante } \\
\text { Themen, über die ich mit anderen } \\
\text { sprechen will. }\end{array}$ & $\begin{array}{l}\text { Eberle et al. (2009) } \\
\text { in Anlehnung an } \\
\text { Prenzel et al. (1996) }\end{array}$ \\
\hline & - & $\begin{array}{l}\text { werde ich oft mit interessanten } \\
\text { Problemen konfrontiert. }\end{array}$ & \\
\hline & - & $\begin{array}{l}\text { interessieren mich die Themen } \\
\text { meistens so sehr, dass ich mich voll } \\
\text { einsetze. }\end{array}$ & \\
\hline \multirow[t]{3}{*}{$\begin{array}{l}\text { Leistungsbezogene } \\
\text { Motivation }\end{array}$} & - & $\begin{array}{l}\text { arbeite ich vor allem deshalb mit, } \\
\text { weil ich immer mein Bestes geben } \\
\text { will. }\end{array}$ & Ramseier (2004) \\
\hline & - & $\begin{array}{l}\text { arbeite ich vor allem deshalb mit, } \\
\text { weil ich einfach überall gut sein } \\
\text { möchte. }\end{array}$ & \\
\hline & - & $\begin{array}{l}\text { arbeite ich vor allem deshalb mit, } \\
\text { weil ich einfach viel leisten will. }\end{array}$ & \\
\hline \multirow[t]{3}{*}{$\begin{array}{l}\text { Langfristig-instrumen- } \\
\text { telle Motivation }\end{array}$} & - & $\begin{array}{l}\text { arbeite ich vor allem deshalb mit, } \\
\text { weil mir das Fach später einmal } \\
\text { nützen wird. }\end{array}$ & Ramseier (2004) \\
\hline & - & $\begin{array}{l}\text { arbeite ich vor allem deshalb mit, } \\
\text { weil das Fach für das, was ich } \\
\text { einmal machen möchte, wichtig } \\
\text { ist. }\end{array}$ & \\
\hline & - & $\begin{array}{l}\text { arbeite ich vor allem deshalb mit, } \\
\text { weil mir das Fach in meinem Beruf } \\
\text { nützen kann. }\end{array}$ & \\
\hline
\end{tabular}

